\title{
Center Maps of Affine Minimal Ruled Hypersurfaces
}

\author{
Masao KATOU \\ Division of Mathematics, Graduate School of Information Sciences, Tohoku University, \\ Aoba, Sendai 980-8579, Japan \\ E-mail:katou@ims.is.tohoku.ac.jp
}

\begin{abstract}
In this paper, we study the center map which is introduced by Furuhata and Vrancken, for affine minimal ruled hypersurfaces.
\end{abstract}

KEYWORDS: Blaschke immersion, affine minimal, ruled surface, affine support function, center map

\section{Introduction}

Affine differential geometry is geometry of submanifolds in the affine space. We study the invariant properties of submanifolds under the group of affine transformations.

In 1908, G. Tzitzéica showed that for a surface in the Euclidean 3-space $\mathbb{R}^{3}$, the ratio of the Gaussian curvature to the fourth power of the support function from the origin $o$ is invariant under all affine transformations fixing $o$, and obtained the notion of an $S$-surface to be a surface for which this ratio is constant. These results of him could be regarded as the beginning of affine differential geometry.

Systematic studies of affine differential geometry have been developed by W. Blaschke, G. Pick, J. Radon, L. Berwald, G. Thomsen, and others during the period from 1916 to 1923, and been studied extensively by T. Kubota, W. Süss, Su Buqing, S. Nakajima and others.

The book of P. A. Schirokow and A. P. Schirokow [8] in 1962 showed remarkable progresses in the local classification of the special classes of surfaces and in the local theory, in particular, the geometry of the induced connections.

In 1982, K. Nomizu formulated the starting point of affine differential geometry from the structual point of view at the Münster Conference. We believe the notion of affine immersion gave a general viewpoint to the subject. And a remarkable textbook [7] by K. Nomizu and T. Sasaki, in 1994 gave systematic introduction, important classical results, and recent developments.

Ruled surfaces in $\mathbb{R}^{3}$ can be visualized by means of wire models and are applied for architecture. These surfaces are not only important for practical use, but also provide artistic inspiration. Thus, the study of ruled surfaces in $\mathbb{R}^{3}$ is an important classical subject in differential geometry and ruled hypersurfaces in higher dimension have also a particular interest. An immersion of an $n$-dimensional manifold into $\mathbb{R}^{n+1}$ is ruled if the manifold admits a continuous codimension one foliation such that the immersion maps each leaf onto an open subset of an affine subspace of $\mathbb{R}^{n+1}$ (cf. for example $[1,4])$.

In [6], we generalized affine minimal ruled surfaces to higher dimension in a manner different from those in [1] and [4]. Indeed, we defined a new notion of ruled hypersurface, which have not been dealt with in affine differential geometry so far.

H. Furuhata and L. Vrancken [3] generalized the center point of a proper affine hypersphere to a map for an affine hypersurface and call it the center map. They investigated the immersions for which the center map is affine congruent with the original hypersurface.

In this paper, we determine completely the center map for some affine minimal ruled hypersurfaces.

I would like to express my gratitude to Professor H. Urakawa for valuable suggestions.

\section{Preliminaries}

Let $M^{n}$ be a smooth manifold and $f: M \rightarrow \mathbb{R}^{n+1}$, a smooth immersion. We can choose a smooth vector field $\xi$ along $f$ which is transversal to $M$, i.e. for all $x \in M$,

$$
T_{f(x)} \mathbb{R}^{n+1}=f_{*}\left(T_{x} M\right) \oplus \mathbb{R} \xi_{x} .
$$

Let $\mathfrak{X}(M)$ be the set of all smooth vector fields on $M$. The canonical connection $D$ on $\mathbb{R}^{n+1}$, induces the torsion free affine connection $\nabla$ and the symmetric $(0,2)$-tensor field $h$ on $M$ with Gauss' formula:

$$
D_{X} f_{*}(Y)=f_{*}\left(\nabla_{X} Y\right)+h(X, Y) \xi
$$

and the (1,1)-tensor field $S$ and the 1-form $\tau$ on $M$ with Weingarten's formula: 


$$
D_{X} \xi=-f_{*}(S X)+\tau(X) \xi
$$

for arbitrary $X, Y \in \mathfrak{X}(M)$.

If $h$ is non-degenerate, $f$ is called a non-degenerate immersion. Then, there is a transversal vector field $\xi$, which is unique up to sign, and satisfies $\tau=0$ and $\theta=\omega_{h}$, where $\omega_{h}$ is the volume form of $h$ and $\theta$ is the volume form on $M$ defined by:

$$
\theta\left(X_{1}, \ldots, X_{n}\right):=\operatorname{det}\left[f_{*}\left(X_{1}\right), \ldots, f_{*}\left(X_{n}\right), \xi\right] .
$$

In this case, $\xi$ is called an affine normal vector field, $f$ with $\xi$ is called a Blaschke immersion, $h$ is called the affine metric, and $(\nabla, h, S)$ is called the Blaschke structure of $f . S$ is called the affine shape operator for $\xi$. In Blaschke structure, it is known that $\nabla S$ is always symmetric. And if $S$ is a constant multiple of the identity, $f: M \rightarrow \mathbb{R}^{n+1}$ is called an affine hypersphere. On a Blaschke immersion, the function defined by $H:=\frac{1}{n} \operatorname{tr} S$ is called the affine mean curvature. Clearly, on an affine hypersphere, $S=H I$. And if $H \equiv 0, f: M \rightarrow \mathbb{R}^{n+1}{ }^{n}$ is called an affine minimal hypersurface.

On a Blaschke immersion, the (0,3)-tensor field $C$ on $M$ defined by $C(X, Y, Z):=\left(\nabla_{X} h\right)(Y, Z)$, called the cubic form, which is totally symmetric. It is known that if $\nabla C$ is totally symmetric then $M$ is an affine hypersphere. And PickBerwald's theorem ( $c f$. [8, p. 53, Theorem 4. 5]) says that $C \equiv 0$ (i.e. $h$ is parallel with respect to $\nabla$ ) if and only if the immersion $f: M \rightarrow \mathbb{R}^{n+1}$ is a quadratic hypersurface.

The function $J$ on $M$ defined by $J:=\frac{1}{4 n(n-1)} h(C, C)$ is called the Pick invariant. It is known that $\hat{\rho}=H+J$, where $\hat{\rho}$ is the scalar curvature of the affine metric $h(c f$. [8, p. 78, Proposition 9. 3]). And in the case $n=2$, the immersion $f$ : $M \rightarrow \mathbb{R}^{3}$ is a ruled surface if and only if $h$ is an indefinite metric and $J \equiv 0(c f$. [8, pp. 89, 90, Definition 11. 1, and Theorems $11.3,11.4]$ ). Hence the surface with $J \equiv 0$ is quadratic or ruled, because definiteness of $h$ and $J \equiv 0$ imply $C \equiv 0$.

If we fix a point $o \in \mathbb{R}^{n+1}$ and call it the origin, we obtain a function $\rho$ on $M$ which is called the affine support function as follows. There exists $Z \in \mathfrak{X}(M)$, for every $x \in M$,

$$
\overrightarrow{o f(x)}=f_{*}\left(Z_{x}\right)+\rho(x) \xi_{x},
$$

where $\overrightarrow{o f(x)}$ is regarded as the element of $T_{f(x)} \mathbb{R}^{n+1}$. Furuhata and Vrancken [3] named the map $c_{f}: M \rightarrow \mathbb{R}^{n+1}$ which is defined as

$$
c_{f}(x):=\overrightarrow{o f(x)}-\rho(x) \xi_{x} \text { for } x \in M
$$

the center map of $f$. Then, they show

Theorem 2.1. [3] $c_{f}$ is an immersion if and only if $\operatorname{ker}(I+\rho S) \cap \operatorname{ker} d \rho=\{0\}$.

In [2], we obtained the following.

Theorem 2.2. (i) Every affine surface with constant Pick invariant in $\mathbb{R}^{3}$ satisfying the condition $R(X, Y) \cdot S=0$ for any vector fields $X$ and $Y$ on $M$ is either an affine sphere with constant curvature metric or an affine minimal ruled surface.

(ii) Every affine minimal ruled surface can be written as $z=y \Psi(x)+\Phi(x)$, where $\Psi(x)$ is a non-constant smooth function in $x$ and $\Phi(x)$ is any smooth function in $x$. Conversely, every surface which can be written as above is an affine minimal ruled surface.

Definition 2.3. We call a hypersurface in $\mathbb{R}^{n+1}$ a ruled hypersurface if it consists of a family of $(n-m)$-dimensional hyperplanes passing through a fixed $m$-dimensional hypersurface. Here, $1 \leq m<n$.

Remark 2.4. Generally, $n$-dimensional ruled hypersurface in the sense of [1] and [4] implies a hypersurface with a foliation by $(n-1)$-dimensional hyperplanes, namely, it is our ruled hypersurface in the case of $m=1$. But we have shown in [6] that it has no Blaschke structure in the case $n \geq 3$. This is the main reason why we consider our ruled hypersurface in general.

From now on, we consider only the case $n=2 m$. In [6], we obtained the following.

Theorem 2.5. Any ruled hypersurface which is written of the form

$$
z=\sum_{k=1}^{m} y^{k} \Psi_{k}\left(x^{1}, \ldots, x^{m}\right)+\Phi\left(x^{1}, \ldots, x^{m}\right),
$$

with $(m+1)$ smooth functions $\Psi_{1}, \ldots, \Psi_{m}, \Phi$ in $\left(x^{1}, \ldots, x^{m}\right)$, is always affine minimal and the Pick invariant $J$ always vanishes, if it is non-degenerate.

Remark 2.6. The necessary and sufficient condition for this hypersurface (1) to be non-degenerate is 


$$
\gamma(x):=\left|\operatorname{det}\left[\frac{\partial \Psi_{i}}{\partial x^{j}}\right]\right|^{\frac{1}{m+1}} \neq 0
$$

Then, the affine normal vector field is

$$
\xi=\left[\begin{array}{c}
0 \\
\vdots \\
0 \\
\gamma(x)
\end{array}\right]-f_{*}\left(\varphi(x) \frac{\partial}{\partial y}\right)
$$

where

$$
\varphi(x):=\left[\frac{\partial \gamma}{\partial x^{1}}, \ldots, \frac{\partial \gamma}{\partial x^{m}}\right]\left[\frac{\partial \Psi_{i}}{\partial x^{j}}\right]^{-1}, \quad \frac{\partial}{\partial y}:=\left[\begin{array}{c}
\frac{\partial}{\partial y^{1}} \\
\vdots \\
\frac{\partial}{\partial y^{m}}
\end{array}\right]
$$

\section{Main Results}

We show the following theorem.

Theorem 3.1. Let

$$
f(x, y)=\left[\begin{array}{c}
x \\
y \\
z
\end{array}\right], \quad x=\left[\begin{array}{c}
x^{1} \\
\vdots \\
x^{m}
\end{array}\right], \quad y=\left[\begin{array}{c}
y^{1} \\
\vdots \\
y^{m}
\end{array}\right],
$$

be a non-degenerate immersion given by (1). And let $o={ }^{t}[0, \ldots, 0] \in \mathbb{R}^{2 m+1}$ be the origin. Then, $c_{f}$ is an immersion. Furthermore, if $c_{f}$ is non-degenerate, it gives an affine minimal ruled hypersurface.

Proof. We obtain with immediate calculation that

$$
\begin{aligned}
S\left(\frac{\partial}{\partial x^{i}}\right) & =\frac{\partial \varphi}{\partial x^{i}} \frac{\partial}{\partial y}, \quad S\left(\frac{\partial}{\partial y^{i}}\right)=0, \quad \text { for } i=1, \ldots, m, \\
\rho & =\frac{1}{\gamma}\left(\Phi-\sum_{l=1}^{m} x^{l}\left(\sum_{k=1}^{m} y^{k} \frac{\partial \Psi_{k}}{\partial x^{l}}+\frac{\partial \Phi}{\partial x^{l}}\right)\right),
\end{aligned}
$$

and

$$
c_{f}=f_{*}\left({ }^{t} x \frac{\partial}{\partial x}+\left({ }^{t} y+\rho \varphi\right) \frac{\partial}{\partial y}\right), \quad \text { where } \quad \frac{\partial}{\partial x}=\left[\begin{array}{c}
\frac{\partial}{\partial x^{1}} \\
\vdots \\
\frac{\partial}{\partial x^{m}}
\end{array}\right] .
$$

If $a \frac{\partial}{\partial x}+b \frac{\partial}{\partial y} \in \operatorname{ker}(I+\rho S)$ for some constants $a=\left[a^{1}, \ldots, a^{m}\right], b=\left[b^{1}, \ldots, b^{m}\right] \in \mathbb{R}^{m}$, then

$$
0=(I+\rho S)\left(a \frac{\partial}{\partial x}+b \frac{\partial}{\partial y}\right)=a \frac{\partial}{\partial x}+\left(b+a \rho \frac{\partial \varphi}{\partial x}\right) \frac{\partial}{\partial y} .
$$

Therefore, we have $a=b=0$, so $\operatorname{ker}(I+\rho S)=\{0\}$. Because of Theorem 2.1, $c_{f}$ is an immersion.

Now, we can write $c_{f}$ as: 


$$
c_{f}(x, y)=\left[\begin{array}{c}
x^{1} \\
\vdots \\
x^{m} \\
y^{1}+\rho \varphi^{1} \\
\vdots \\
y^{m}+\rho \varphi^{m} \\
\sum_{k=1}^{m}\left(y^{k}+\rho \varphi^{k}\right) \Psi_{k}+\sum_{l=1}^{m} x^{l}\left(\sum_{k=1}^{m} y^{k} \frac{\partial \Psi_{k}}{\partial x^{l}}+\frac{\partial \Phi}{\partial x^{l}}\right)
\end{array}\right]=:\left[\begin{array}{c}
x^{1} \\
\vdots \\
\tilde{x^{m}} \\
\widetilde{y^{1}} \\
\vdots \\
\tilde{y^{m}} \\
\widetilde{z}
\end{array}\right]
$$

where $\varphi=:\left[\varphi^{1}, \ldots, \varphi^{m}\right]$. Because of $(5), \rho$ can be considered as a linear function in $y^{1}, \ldots, y^{m}$, so can $\widetilde{y^{1}}, \ldots, \widetilde{y^{m}}, \widetilde{z}$. Therefore, with exchange of base from $\left\{x^{1}, \ldots, x^{m}, y^{1}, \ldots, y^{m}\right\}$ to $\left\{\widetilde{x}^{1}, \ldots, \widetilde{x^{m}}, \widetilde{y^{1}}, \ldots, \widetilde{y^{m}}\right\}, \widetilde{z}$ can be considered as a linear function in $\widetilde{y^{1}}, \ldots, \widetilde{y^{m}}$, whose coefficients are functions in $\widetilde{x}^{1}, \ldots, \widetilde{x^{m}}$. (If the exchange of base is impossible then $c_{f}$ must not be non-degenerate.) I.e., it can be written of the form

$$
\widetilde{z}=\sum_{k=1}^{m} \widetilde{y^{k}} \widetilde{\Psi_{k}}\left(\widetilde{x^{1}}, \ldots, \widetilde{x^{m}}\right)+\widetilde{\Phi}\left(\widetilde{x^{1}}, \ldots, \widetilde{x^{m}}\right) .
$$

Then, because of Theorem 2.5, if $c_{f}$ is a non-degenerate immersion, it is an affine minimal ruled hypersurface. Hence we obtain Theorem 3.1.

Remark 3.2. Because of (2), $c_{f}$ is non-degenerate if and only if $\operatorname{det}\left[\frac{\partial \tilde{\Psi}_{i}}{\partial x^{j}}\right] \neq 0$.

Example 3.3. We consider the case $m=1$. We have seen at Theorem 2.2 that every affine minimal ruled surface in $\mathbb{R}^{3}$ can be written as $z=y \Psi(x)+\Phi(x)$. The necessary and sufficient condition for it to be non-degenerate is that $\Psi$ is not a constant. Because of (3), the affine normal vector field is

$$
\xi=\left[\begin{array}{l}
0 \\
0 \\
\gamma
\end{array}\right]-f_{*}\left(\varphi \frac{\partial}{\partial y}\right)=\left[\begin{array}{c}
0 \\
\pm\left(\left|\Psi^{\prime}\right|^{-\frac{1}{2}}\right)^{\prime} \\
\pm\left(\left|\Psi^{\prime}\right|^{-\frac{1}{2}} \Psi\right)^{\prime}
\end{array}\right]
$$

where $\gamma=\left|\Psi^{\prime}\right|^{\frac{1}{2}}, \varphi=\mp\left(\left|\Psi^{\prime}\right|^{-\frac{1}{2}}\right)^{\prime}$. Then, we have $\rho=\left|\Psi^{\prime}\right|^{-\frac{1}{2}}\left(\Phi-x\left(y \Psi^{\prime}+\Phi^{\prime}\right)\right)$ and

$$
\begin{aligned}
c_{f}(x, y) & =\left[\begin{array}{c}
x \\
y\left(1-\frac{1}{2} x\left(\log \left|\Psi^{\prime}\right|\right)^{\prime}\right)-\frac{1}{2}\left(\frac{1}{\Psi^{\prime}}\right)^{\prime}\left(\Phi-x \Phi^{\prime}\right) \\
y\left(\Psi+x\left(\left|\Psi^{\prime}\right|^{-\frac{1}{2}} \Psi\right)^{\prime}\left|\Psi^{\prime}\right| \frac{1}{2}\right) \pm x \Phi^{\prime}\left(\left|\Psi^{\prime}\right|^{-\frac{1}{2}} \Psi\right)^{\prime}\left|\Psi^{\prime}\right|^{-\frac{1}{2}}-\frac{1}{2}\left(\frac{1}{\Psi^{\prime}}\right)^{\prime} \Psi \Phi
\end{array}\right] \\
= & {\left[\begin{array}{c}
\tilde{x} \\
\tilde{y} \\
\tilde{y}\left(\Psi-\frac{\Psi^{\prime}}{\left(\log \left|\Psi^{\prime} / \tilde{x}^{2}\right|\right)^{\prime}}\right)-\frac{\tilde{x}^{2}\left(\log \left|\Psi^{\prime}\right|\right)^{\prime}(\Phi / \widetilde{x})^{\prime}}{2\left(\log \left|\Psi^{\prime} / \tilde{x}^{2}\right|\right)^{\prime}}+\widetilde{x} \Phi^{\prime}
\end{array}\right] . }
\end{aligned}
$$

Therefore, if $\Psi-\frac{\Psi^{\prime}}{\left(\log \left|\Psi^{\prime} / \tilde{x}^{2}\right|\right)^{\prime}}$ is not a constant, $c_{f}$ is an affine minimal ruled surface.

\section{REFERENCES}

[1] Barbosa, J. L. M., and Delgado, J. A., "Ruled submanifolds of space forms with mean curvature of nonzero constant length," Amer. J. Math., 106: 763-780 (1984).

[2] Belkhelfa, M., and Katou, M., "Affine semiparallel surfaces with constant Pick invariant," Hokkaido Math. J., 34: 355-374 (2005).

[3] Furuhata, H., and Vrancken, L., "The center map of an affine immersion," Hokkaido University preprint series in mathematics, series \# 665 (2004).

[4] Hájková, V., and Kowalski, O., "Intrinsic characterization of completely ruled hypersurfaces," Publ. Math. Debrecen, 54: 5562 (1999).

[5] Jelonek, W., "Affine surfaces with parallel shape operators," Ann. Polon. Math., 56: 179-186 (1992).

[6] Katou, M., "New affine minimal ruled hypersurfaces," to appear in J. Math. Soc. Japan.

[7] Nomizu, K., and Sasaki, T., Affine Differential Geometry: Geometry of Affine Immersions, Cambridge University Press, (1994).

[8] Schirokov, P. A., and Schirokov, A. P., Affine Differentialgeometrie, Teubner, Leibzig (1962). 